\title{
Theoretical Investigation on Charge Transfer Properties of 1,3,5-Tripyrrolebenzene (TPB) and its Derivatives with Electron-withdrawing Substituents
}

\author{
Yong Hu, Jun Yin, Kadali Chaitanya, Xue-Hai Ju*
}

\author{
Key Laboratory of Soft Chemistry and Functional Materials of MOE, School of Chemical Engineering, Nanjing University of Science and Technology, Nanjing \\ 210094, P. R. China \\ * Corresponding author's e-mail address: xhju@njust.edu.cn
}

RECEIVED: November 9, $2015 *$ REVISED: March 3, 2016 * ACCEPTED: March 14, 2016

\begin{abstract}
The electronic structures and charge transport properties of 1,3,5-tripyrrolebenzene (TPB) and its substituted derivatives with $-\mathrm{F}$ and $-\mathrm{CN}$ groups have been investigated by DFT calculations in combination with the Marcus hopping model. The dimer geometry was optimized by density functional theory method with dispersion force correction being included (DFT-D). Consequently, the charge transfer integral was evaluated. The calculation results show that the introduction of electron-withdrawing substituents does not significantly change the bond lengths and molecular symmetry of TPB, but lower the coplanarity between the pyrrole and benzene rings, especially in the case of $\mathrm{CN}$ substitution. Meanwhile, the introduction of electron-withdrawing groups can decrease the energy of the frontier molecular orbital and enhance the air stability. Fluorination makes the $\lambda_{\mathrm{e}}$ increase obviously while cyanation dose not. Generally speaking, the $\lambda_{e}$ values of the title compounds are larger than their $\lambda_{\mathrm{h}}$. Except for compounds 6 and 9 , all others keep the face to face packing or have a slight slip in dimers, but the center of mass distances increase after fluorination or cyanation due to the distortion of the monomer's coplanarity. The predicted quasi-one-dimensional electron mobility of the dimers is up to $0.433 \mathrm{~cm} \cdot \mathrm{V}^{-1} \cdot \mathrm{s}^{-1}$ at $298.15 \mathrm{~K}$. The electron injection barriers of 2 and 7 are lower than that of TPB. The TPB derivatives of 1, 2, and 7 are potential $n$-channel materials with the high electron mobility.
\end{abstract}

Keywords: 1,3,5-tripyrrolebenzene (TPB) and its derivatives, fluorination and cyanation, DFT, Marcus-Hush theory, organic semiconductor, charge mobility.

\section{INTRODUCTION}

A significant progress has been made on the field of organic semiconductor (OSC) during the last several decades. ${ }^{[1-4]}$ The charge mobility of several kinds of OSC is now as large as $10 \mathrm{~cm} \cdot \mathrm{V}^{-1} \cdot \mathrm{s}^{-1}$ in the thin films and single crystals ${ }^{[5-10]}$ such as pentacene. But there are still some unsolved problems. The development of $n$-type OSC falls behind the p-type in the aspects of the charge mobility and the environment stability, The most obvious difference of these two kinds of OSC is that the mobility of high-performance $p$-type can be as high as amorphous silicon while most $n$-type OSC are less than $1 \mathrm{~cm}^{2} \cdot \mathrm{V}^{-1} \cdot \mathrm{s}^{-1}$. This unbalanced development of OSC brings a heavy negative effect on its application especially on the devices such as complementary integrated circuits, organic $p-n$ junctions, and bipolar transistors. Hence, it is a hot topic to search for high-performance stable $n$-type OSC.

1,3,5-tripyrrolebenzene (TPB) contains a benzene ring and three pyrrole rings that connect to benzene ring by the $\mathrm{C}-\mathrm{N}$ single bond, as shown in Figure 1 . Its synthesis can be dated back to last 1990s. [11] The central benzene rings stack face to face with a close distance (as short as $3.34 \AA$ ) and show a perfect $\pi-\pi$ overlap in spatial along the crystallographic c-axis. The TPB crystal structure is depicted in Figure 1(c). These features forebode a potential high-performance OSCs. Though the molecule was synthesized and the crystal structure was determined more than ten years ago, TPB was considered as potential OSC materials in 2013 by Brett Ellman and Robert J. 


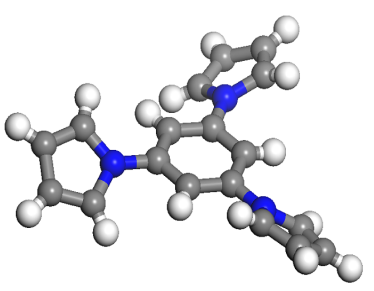

(a)

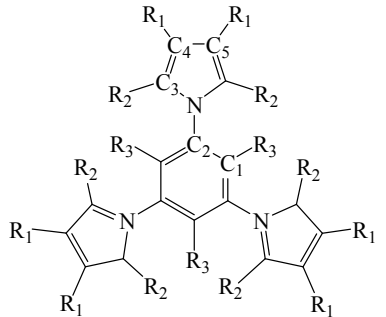

(b)

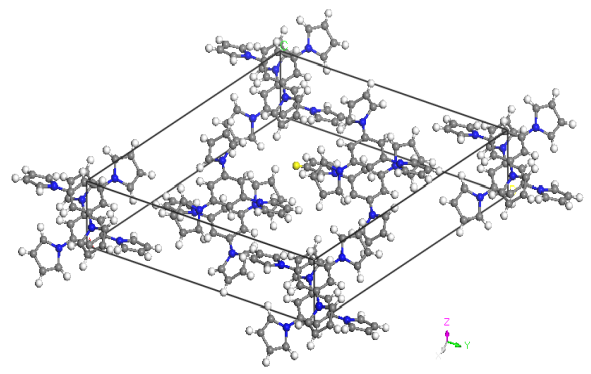

(c)

Figure 1. (a) Molecular structure, (b) positions of $R_{1}, R_{2}$ and $R_{3}$ substituents and (c) crystal structure of TPB.

Twieg. ${ }^{[12]}$ They have studied TPB from the aspect of band structure and its unusual packing by density functional theory (DFT). The outcomes show that it has very large $\pi$ overlap in the benzene stacks, broad bands, and relatively small binding energies, making TPB a promising quasi-one dimensional electron-transport material. However, the charge mobility properties of TPB single crystal have not been investigated. What's more, considering the excellent $\pi$-overlap in the crystal, some electronic-withdrawing groups could be attached to the aromatic rings while keeping its quasi one-dimensional $\pi$-stack mode. Up to now, fluoro-, perfluoroalkyl-, cyano- and percyano-TPB have not been reported. In this work, we calculated the molecular geometry, electronic structure, dimer packing and charge transfer mobility of TPB and its derivatives, and analyzed the influence of electron-drawing groups on the electron transfer properties. In order to keep the symmetry of these molecules, the substituent groups were placed in symmetrical position. Thus eight new molecules (2-9) were constructed as shown in Table 1 . This study is to find new materials and add some valuable information for improving charge transfer mobility together with searching or designing new promising n-type OSC. The molecular structures of TBP substituted derivatives are depicted in Figure 1(b).

Table 1. Numbering of Substituted TPB.

\begin{tabular}{cccccccc}
\hline Compd. & $\mathrm{R}_{1}$ & $\mathrm{R}_{2}$ & $\mathrm{R}_{3}$ & Compds & $\mathrm{R}_{1}$ & $\mathrm{R}_{2}$ & $\mathrm{R}_{3}$ \\
\hline 1 & $\mathrm{H}$ & $\mathrm{H}$ & $\mathrm{H}$ & 6 & $\mathrm{CN}$ & $\mathrm{H}$ & $\mathrm{H}$ \\
2 & $\mathrm{~F}$ & $\mathrm{H}$ & $\mathrm{H}$ & 7 & $\mathrm{H}$ & $\mathrm{CN}$ & $\mathrm{H}$ \\
3 & $\mathrm{H}$ & $\mathrm{F}$ & $\mathrm{H}$ & 8 & $\mathrm{H}$ & $\mathrm{H}$ & $\mathrm{CN}$ \\
4 & $\mathrm{H}$ & $\mathrm{H}$ & $\mathrm{F}$ & 9 & $\mathrm{CN}$ & $\mathrm{H}$ & $\mathrm{CN}$ \\
5 & $\mathrm{~F}$ & $\mathrm{H}$ & $\mathrm{F}$ & & & & \\
\hline
\end{tabular}

\section{THEORETICAL MODELS AND COMPUTATIONAL DETAILS}

\section{Theoretical Models}

Coherent band model[13-14] and incoherent hopping model ${ }^{[15-16]}$ are the two classical theoretical models in describing the charge transport process in the solid materials. The coherent band model was usually applied in system with strong interaction between two neighbor molecules while the incoherent hopping model was usually applied to deal with intermolecular interactions of which most are non-covalent interactions such as hydrogen bonding, $\pi$-stacking, polar-nonpolar. Thus, the hopping model is more suitable for investigating the charge transfer process between adjacent molecules in OSCs. ${ }^{[17,18]}$ In the Marcus-Hush theory (hopping model) ${ }^{[19-21]}$, the hopping rate (or the charge transfer rate) $k$ can be described as

$$
k=\frac{H_{\text {eff }}^{2}}{\hbar}\left(\frac{\pi}{\lambda k_{B} T}\right)^{1 / 2} \exp \left(-\frac{\lambda}{4 k_{B} T}\right)
$$

Here the $\lambda$ is the reorganization energy. In theory, $\lambda=\lambda_{\text {int }}+$ $\lambda_{\text {ext }}$. But the $\lambda$ was often replaced by inner reorganization energy $\lambda_{\text {int, }}$, because the external reorganization energy $\lambda_{\text {ext }}$ has been verified to be very small. ${ }^{[22-24]} H_{\text {eff }}$ is the effective charge transfer integral between adjacent molecules, $H_{\text {eff, }}$ $T, \hbar$ and $k_{B}$ are the effective charge transfer integral, the absolute temperature, the reduced Planck constant and Boltzmann constant, respectively. Then the charge diffusion coefficient $(D)$ is given by:

$$
D=\frac{1}{2 n} \sum r_{i}^{2} k_{i} p_{i}
$$


Here, $n$ means the dimensionality which is equal to 1 for this quasi-one-dimensional TPB crystal in this work, $k_{i}$ is hopping rate of the ith hopping pathway while $r_{i}$ is the hopping distance between the adjacent molecules. $p_{i}$ is the relative probability for the $i$ th hopping pathway, $p_{i}=k_{i} / \sum k_{j}$. The $\lambda_{\text {int }}$ reflects the potential energy changes during the charge transfer process. ${ }^{[25]}$ Usually, $\lambda_{\text {int }}$ is defined as:

$$
\lambda=\left(E_{ \pm}^{*}-E_{ \pm}\right)+\left(E^{*}-E\right)
$$

where $E$ and $E_{ \pm}$are the energy of neutral and cation/anion, $E^{*}$ represents the energy of cation or anion in neutral molecule geometry, and $E_{ \pm}^{*}$ means the energy of neutral molecule in cationic or anionic geometry. The reorganization energy should be as small as possible to achieve a high charge mobility $(\mu) .^{[26-31]}$

$H_{\text {eff }}$ reflects the electronic coupling intensity between the adjacent molecules and playing a vital role in determining the value of $\mu$. It can be calculated as follows:

$$
\begin{aligned}
& H_{\text {eff }}=\frac{H-S \cdot\left(E_{1}+E_{2}\right) / 2}{1-S^{2}} \\
& H=\left\langle\Phi_{\text {HOMO/LUMO }}^{0, \text { site 1 }}\left|F^{0}\right| \Phi_{\text {HOMO/LUMO }}^{0, \text { site2 }}\right\rangle \\
& F^{0}=S \cdot C \cdot \varepsilon \cdot C^{-1}
\end{aligned}
$$

$H$ means the charge transfer integral which is defined in Eq (5). $E_{i}(i=1,2)$ and $S$ are the site energy of frontier molecular orbital for monomer and the overlap matrix for the dimer, respectively. In (5), $\Phi$ represents the frontier molecular orbital while $F^{0}$ is the unperturbed Fock operator for the specific dimer which can be evaluated by Eq (6). The Kohn-Sham orbital $C$ and eigenvalue $\varepsilon$ are obtained by diagonalizing the zeroth-order Fock matrix without any selfconsistent field iteration. This direct method has been widely employed. ${ }^{[32]}$ Actually, the $H_{\text {eff }}$ heavily depends on the relative packing and only the nearest or the best face to face dimers which contribute the most effective charge transport were taken into account in evaluating $\mu$ in many works. ${ }^{[33]}$ According to Einstein relation, charge mobility $(\mu)$ can be evaluated by ${ }^{[34,35]}$ :

$$
\mu=e D / k_{B} T
$$

where $e$ represents the electronic charge.

\section{Computational Details}

As we all known that DFT is a good formalism to calculate the molecular geometry and electronic properties of organic compounds as shown by many former works. ${ }^{[36-38]}$ The studied TPB derivatives have not been synthesized, and their geometries are lacked. The molecular structures of the series of TPB and derivatives are optimized by the DFTB3LYP/6-311++G** [30] method. Then, the $\lambda$ (for both electron and hole) values, as well as the ionization potentials and electron affinities were obtained at the same computational level. ${ }^{[26,29]}$ In addition, the HOMO/LUMO energy were estimated by O3LYP, PBE, B3LYP, and TPSSh functionals with $6-311 \mathrm{G} *$ basis set. ${ }^{[39-43]}$

All the stacking motifs of studied compounds from 2 to $\mathbf{9}$ are unknown. Considering the fact that the molecular structures of our studied compounds are similar, it is rational to assume that TPB and its derivatives have similar face to face molecular packing way between the adjacent molecules. The corresponding position $\mathrm{H}$ atoms were replaced by $-\mathrm{F}$ or $-\mathrm{CN}$ group on the basis of TPB face to face dimer, and the dimers were optimized at $\omega B 97 X D / 6-31 G$ level. ${ }^{[25]}$ The $H_{\text {eff }}$ can be estimated on the basis of the predicted dimers coupling with fragment orbital approach for the neighbor molecular pair. The calculations of $H_{\text {eff }}$ were implemented by the PW91PW91/6-31G* ${ }^{[44-45]}$ in the AOMIX program. ${ }^{[46]}$ All the quantum-chemical computations were performed using the Gaussian 09 program. ${ }^{[47]}$

\section{RESULTS AND DISCUSSION}

\section{Geometries of TPB and Derivatives}

Table 2 lists all the optimized bond lengths and the dihedral angel between pyrrole and benzene rings of TPB and its derivatives at the B3LYP/6-311++G** level. And the experimental value of TPB molecule was also shown. It should be noted that the pyrrole ring inclines at a dihedral angle of $\theta$ to the plane of the central phenyl ring. There is no doubt that $\theta$ will change upon different $\mathrm{R}_{1}, \mathrm{R}_{2}$ and $\mathrm{R}_{3}$ groups. In Table 2, the bond length and $\theta$ of TPB from simulation and experiment were compared. It can be seen that the bond lengths agree well with each other. The largest deviation between them is only $0.012 \AA$ except for $C_{1}-R_{3}, C_{3}-R_{2}, C_{4}-R_{1}$ bond. In 1 , the difference between the experimental and calculated values of $\mathrm{C}-\mathrm{H}$ bond length is larger than other bonds such as $\mathrm{C}=\mathrm{C}, \mathrm{C}-\mathrm{C}$ or $\mathrm{C}-\mathrm{N}$ bond. In general, the bond length do not have obvious difference, the largest fluctuation of $\mathrm{C}_{1}=\mathrm{C}_{2}, \mathrm{C}_{2}-\mathrm{N}, \mathrm{N}-\mathrm{C}_{3}, \mathrm{C}_{3}=\mathrm{C}_{4}$ and $\mathrm{C}_{4}-$ $\mathrm{C}_{5}$ bonds are less than $0.03 \AA$. Among them, $\mathrm{C}_{1}=\mathrm{C}_{2}$ and $\mathrm{N}-$ $\mathrm{C}_{3}$ almost keep the same especially in $\mathbf{1}$ to $\mathbf{5}$. The bond lengths of $\mathrm{N}-\mathrm{C}_{3}, \mathrm{C}_{3}-\mathrm{C}_{4}$ and $\mathrm{C}_{4}-\mathrm{C}_{5}$ which are within or connect to the pyrrole ring are averaged. This means that the pyrrole rings in $\mathbf{7}$ are more conjugated than those in the others, which may contribute to $\lambda_{\mathrm{e}}$ decreasing. 
Table 2. Optimized geometries of title compounds at B3LYP/6-311++G** level. (a)

\begin{tabular}{ccccccccccc}
\hline Compd. & $\mathrm{C}_{1}=\mathrm{C}_{2}$ & $\mathrm{C}_{2}-\mathrm{N}$ & $\mathrm{N}-\mathrm{C}_{3}$ & $\mathrm{C}_{3}-\mathrm{R}_{2}$ & $\mathrm{C}_{3}=\mathrm{C} 4$ & $\mathrm{C}_{4}-\mathrm{R}_{1}$ & $\mathrm{C}_{4}-\mathrm{C}_{5}$ & $\mathrm{C}_{1}-\mathrm{R}_{3}$ & $\theta /^{\circ}$ \\
\hline $1^{(\mathrm{b})}$ & 1.389 & 1.416 & 1.385 & 0.9291 & 1.361 & 0.9560 & 1.416 & 0.9301 & 31.58 \\
1 & 1.396 & 1.416 & 1.385 & 1.077 & 1.373 & 1.079 & 1.426 & 1.081 & 35.62 \\
$\mathbf{2}$ & 1.395 & 1.415 & 1.386 & 1.075 & 1.370 & 1.337 & 1.415 & 1.081 & 34.31 \\
$\mathbf{3}$ & 1.394 & 1.423 & 1.387 & 1.335 & 1.357 & 1.077 & 1.440 & 1.080 & 46.90 \\
$\mathbf{4}$ & 1.397 & 1.409 & 1.388 & 1.077 & 1.370 & 1.079 & 1.428 & 1.339 & 55.34 \\
$\mathbf{5}$ & 1.400 & 1.409 & 1.391 & 1.077 & 1.371 & 1.342 & 1.419 & 1.343 & 54.37 \\
$\mathbf{6}$ & 1.394 & 1.421 & 1.378 & 1.077 & 1.377 & 1.417 & 1.441 & 1.082 & 40.32 \\
7 & 1.393 & 1.429 & 1.388 & 1.413 & 1.387 & 1.078 & 1.404 & 1.081 & 51.86 \\
8 & 1.410 & 1.404 & 1.394 & 1.077 & 1.366 & 1.079 & 1.429 & 1.430 & 56.40 \\
\hline
\end{tabular}

(a) Bond length in $\AA$, dihedral angle between pyrrole and benzene rings in degrees.

(b) Experimental X-ray crystal structure.

The $\theta$ values were listed in Table 2. The deviation between calculated and experimental values of $\theta$ for TPB is $4.04^{\circ}$ which is not neglectable. The $\theta$ values for TPB by using $6-31 \mathrm{G}^{* *}, 6-311 \mathrm{G}^{*}$ and $6-311++\mathrm{G}^{* *}$ basis sets are $34.79^{\circ}$, $36.20^{\circ}$ and $35.62^{\circ}$ respectively. The use of different basis sets cannot explain the discrepancy of $4.04^{\circ}$ totally. The main reason is that the intermolecular interactions in crystal result in a slight change of molecular geometries in comparison with its gaseous state. There is no doubt that the location and number of substituent groups have an influence on the molecular geometry. All substituent make the $\theta$ become larger except for $\mathbf{2}$. This means that $\mathbf{2}$ have a better planarity and the face to face dimer of $\mathbf{2}$ may have a shorter center-of-mass distance than others. From 2 to $\mathbf{4}$, the $\theta$ value increases obviously as $-\mathrm{F}$ moved from $\mathrm{R}_{1}$ to $\mathrm{R}_{3}$ site. The same trend is also true in $\mathbf{6 , 7}$ and $\mathbf{8}$. These can attribute to the steric hindrance and inner stress. The $\theta$ value has an increasing trend as number of substituent groups increases especially in $R_{2}$ and $R_{3}$. Both $\mathbf{2}$ and $\mathbf{6}$ are substituted in $\mathrm{R}_{1}$ site. It should be noticed that the $\theta$ is obvious larger in the case of $-\mathrm{CN}$ substituent than $-\mathrm{F}$. The $-\mathrm{CN}$ has a larger influence than $-\mathrm{F}$, not only in bond length but also in $\theta$, due to its larger size than $-\mathrm{F}$. From this perspective, both $-\mathrm{F}$ and $-\mathrm{CN}$ substituents in $\mathrm{R}_{2}$ or $\mathrm{R}_{3}$ position is not beneficial to the molecular coplanarity.

Table 3. Calculated energies of HOMOs, LUMOs and their gap for TPB by using different functionals with 6-311G* basis set. (a) $^{\text {(a) }}$

\begin{tabular}{cccc}
\hline Functional & HOMO & LUMO & gap \\
\hline PBEPBE & -5.40 & -1.95 & 3.45 \\
B3LYP & -6.13 & -1.10 & 5.03 \\
O3LYP & -5.65 & -1.25 & $4.40(4.20)$ \\
TPSSh & -5.73 & -1.33 & 4.40 \\
\hline
\end{tabular}

(a) Energy in eV. Experimental value in parenthesis.

\section{Frontier Molecular Orbital, Ionization Potentials, Electron Affinities and Reorganization Energies}

In order to select a suitable functional for the frontier molecular orbital simulation, B3LYP, O3LYP, PBEPBE and TPSSh functional are tested for TPB. The outcomes were listed in Table 3. Ellman et al. has measured the ultraviolet spectrum in vapor phase of TPB, and obtained an experimental optical bandgap of $4.2 \mathrm{eV} \cdot{ }^{[13]}$ This offers a good reference to choose functional. The outcome shows that O3LYP and TPSSh produce an reasonable gap energy in comparison with the experiment. The calculated energies of HOMOs and LUMOs together with HOMO-LUMO gaps for TPB and its derivatives at the O3LYP (TPSSh)/6-311G* level were listed in Table 4. The contours of their frontier molecular orbitals were showed in Figure 2. In general,

Table 4. Calculated energies of HOMOs and LUMOs and their gaps.

\begin{tabular}{|c|c|c|c|c|c|c|}
\hline \multirow[b]{2}{*}{ Compd. } & \multicolumn{3}{|c|}{ O3LYP/6-311G* } & \multicolumn{3}{|c|}{ TPSSh/6-311G* } \\
\hline & $E_{\text {LUMO }}$ & Еномо & $E_{\mathrm{g}}$ & $E_{\text {LUMO }}$ & Еномо & $E_{\mathrm{g}}$ \\
\hline 1 & -1.25 & -5.65 & 4.40 & -1.33 & -5.73 & 4.40 \\
\hline 2 & -1.87 & -6.23 & 4.36 & -1.91 & -6.29 & 4.38 \\
\hline 3 & -1.58 & -5.55 & 3.97 & -1.64 & -5.62 & 3.98 \\
\hline 4 & -1.51 & -5.68 & 4.18 & -1.57 & -5.76 & 4.18 \\
\hline 5 & -2.08 & -6.23 & 4.15 & -2.17 & -6.35 & 4.18 \\
\hline 6 & -3.32 & -7.62 & 4.30 & -3.44 & -7.72 & 4.28 \\
\hline 7 & -2.80 & -6.96 & 4.17 & -2.76 & -6.97 & 4.20 \\
\hline 8 & -3.25 & -6.05 & 2.80 & -3.25 & -6.12 & 2.87 \\
\hline 9 & -5.03 & -7.88 & 2.86 & -5.06 & -7.96 & 2.90 \\
\hline
\end{tabular}




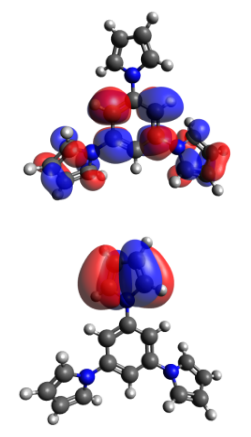

1
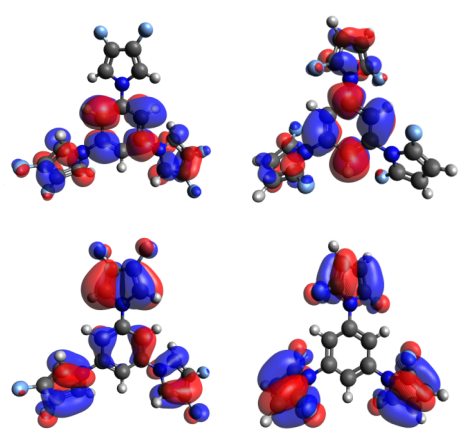

2

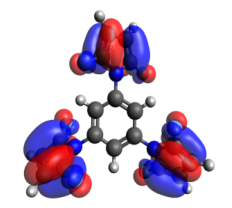

3
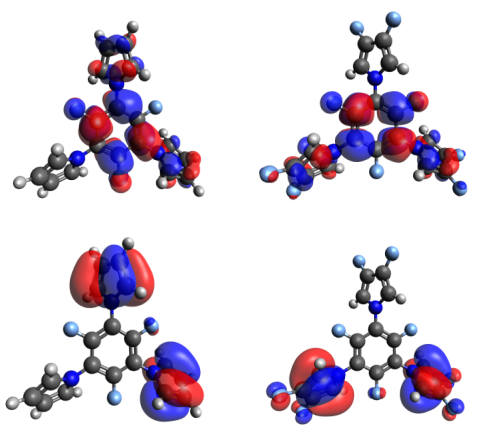

4

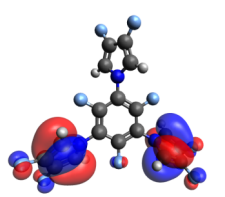

5

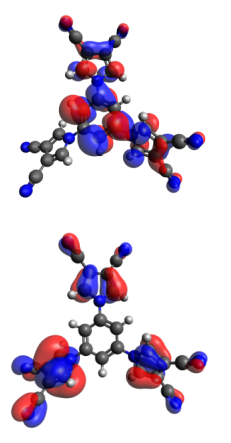

6
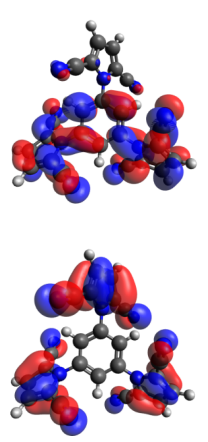

7
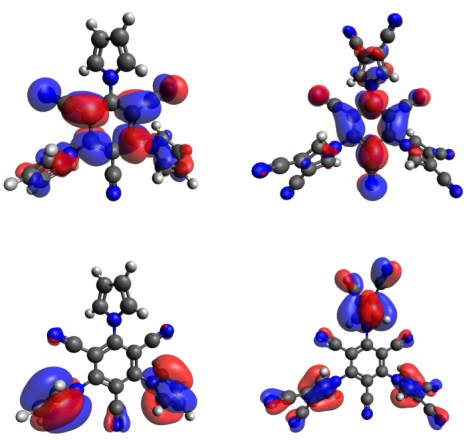

8

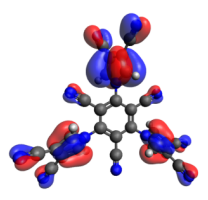

9

Figure 2. Representations of LUMOs (up) and HOMOs (down) at B3LYP/6-311G* level.

most HOMOs center in the pyrrole rings while the LUMOs distribute in both peripheral pyrrole rings and the central phenyl ring. The contribution of outside three pyrrole rings to LUMO of 1, 4 and 8 are $32.84 \%, 19.61 \%$ and $14.53 \%$, respectively. This means that when attaching $-\mathrm{F}$ groups to the pyrrole rings, the LUMOs distribute more on the central phenyl ring. And $-\mathrm{CN}$ substituents aggravate this trend even though the $-\mathrm{CN}$ and $-\mathrm{F}$ also make some atomic orbital contribution to LUMO. At the same time, it can be found that the larger the $\theta$ is, the less the LUMO distributes in peripheral pyrrole rings and more in central benzene rings for $\mathbf{2}, \mathbf{3}, \mathbf{4}$ and $\mathbf{6}, \mathbf{7}, \mathbf{8}$ and $\mathbf{9}$. The $\boldsymbol{\theta}$ of cyanated derivatives is generally larger than the fluorinated ones. When the substituent groups are in $\mathrm{R}_{3}$ position, the LUMO distributes more in the central ring. While the groups are at $R_{1}$ or $R_{2}$ positions, the HOMO distributes more in the outside rings. No matter what site these groups are attached to, the frontier orbitals will extend to the groups. This means that electron withdrawing groups have a great influence on the frontier molecular orbital. The HOMO/LUMO energy level in Table 4 shows that the two functionals used produce very similar outcome. The LUMO of $\mathbf{1}$ is $-1.25(-1.33) \mathrm{eV}$, much higher than Au work function $(-5.1 \mathrm{eV})$. While the LUMOs of all the other substituted compounds decrease somewhat, especially for cyano substitutions. These results show that the attachment of $-\mathrm{F}$ and $-\mathrm{CN}$ groups to TPB not only stabilizes the frontier molecular orbital remarkably and then lowers the electron injection barrier, but also enhances the ambient stability. This is in agreement with the previous study. ${ }^{[48-50]}$ At the same time, the energy gap also decreases mainly due to the decreasing of LUMO energy.

Table 5 shows the bond-length change $(\Delta d)$ of the studied derivatives during the electron transport process. Here, the $\Delta d_{i}$ can be defined as the difference of the bond length between the optimized anion and neutral molecules. The $C_{1}-C_{2}, C_{2}-N$ and $C_{1}-R_{3}$ bonds of most compounds always have the largest bond-length change. These bonds belong to or adjacent to the central conjugated benzene ring. This means that when an electron is attached upon these molecules, the benzene ring will be affected. These are in agreement with the LUMO distributions as shown in Figure 2. The $\mathrm{C}-\mathrm{H}$ bonds always have a small length change during the process for all derivatives. The bond-length changes $(\Sigma|\Delta r|)$ from 1 to 9 were summed up as follow: $0.058 \AA, 0.097 \AA, 0.057 \AA, 0.11 \AA$, $0.10 \AA, 0.033 \AA, 0.12 \AA, 0.053 \AA$ and $0.063 \AA$, respectively. The fluorine substituted derivatives have a larger $\Sigma|\Delta r|$ value than cyanated analogs except for $\mathbf{3}$ and $\mathbf{7}$. 
Table 5. Bond-length changes ( $\Delta d$ in $\AA$ ) upon electron transport process at B3LYP/6-311G** level

\begin{tabular}{cccccccccc}
\hline compd. & $\Delta d_{\mathrm{C} 1=\mathrm{C} 2}$ & $\Delta d_{\mathrm{C} 2 \mathrm{~N}}$ & $\Delta d_{\mathrm{N}-\mathrm{C} 3}$ & $\Delta d_{\mathrm{C} 3-\mathrm{R} 2}$ & $\Delta d_{\mathrm{C} 3=\mathrm{C} 4}$ & $\Delta d_{\mathrm{C} 4 \mathrm{R} 1}$ & $\Delta d_{\mathrm{C} 4-\mathrm{C} 5}$ & $\Delta d_{\mathrm{C} 1 \mathrm{R} 3}$ \\
\hline 1 & -0.019 & 0.016 & -0.007 & 0.000 & 0.008 & 0.001 & -0.004 & 0.001 \\
2 & 0.047 & 0.002 & -0.003 & -0.000 & 0.013 & 0.016 & -0.014 & -0.001 \\
3 & -0.005 & 0.027 & -0.014 & 0.001 & 0.008 & 0.000 & -0.001 & 0.001 \\
4 & 0.038 & 0.013 & -0.008 & -0.000 & 0.008 & 0.001 & -0.003 & 0.038 \\
5 & 0.032 & 0.009 & -0.008 & 0.000 & 0.007 & 0.015 & -0.008 & 0.021 \\
6 & -0.002 & -0.009 & 0.002 & -0.001 & 0.009 & -0.003 & -0.006 & 0.001 \\
7 & 0.018 & -0.026 & 0.020 & -0.013 & 0.020 & 0.000 & -0.018 & -0.001 \\
8 & -0.003 & 0.016 & -0.010 & -0.000 & 0.005 & 0.001 & -0.002 & -0.016 \\
9 & 0.028 & 0.012 & -0.010 & 0.000 & 0.006 & -0.001 & -0.002 & -0.003 \\
\hline
\end{tabular}

The harmonic oscillator model assumed in Marcus theory has been applied widely and shown to be an effective method to estimate $\lambda^{\left[{ }^{[51]}\right.}$ In this work, a series of basis sets have been tested to evaluate the influence of basis sets to ionization potentials $(I P)$, electron affinities $(E A)$, and reorganization energies ( $\lambda_{\mathrm{h}}$ and $\lambda_{\mathrm{e}}$ ) of $\mathbf{1}$ (Table 6$)$. In this work, vertical $I P_{v}\left(E A_{v}\right)$ were the difference between the energy of cationic (anionic) in the neutral geometry and the energy of neutral species. While the adiabatic $I P_{a}\left(E A_{a}\right)$ were defined as the difference between the relaxed cationic (anionic) and the neutral molecule. It can be seen that the variations of $I P$ and $\lambda$ are small while the $E A$ has an obviously decrease as the basis sets become larger. The trend of $E A$ is caused by the incomplete basis set which has been particularly studied by Ramírez-Solís and his coworker ${ }^{[52]}$. Given this, the values of $E A$ in Table 6 offer a relative and intercomparable outcome. In Table 6, the $\lambda_{\mathrm{e}}$ decreases from $0.306 \mathrm{eV}$ to $0.285 \mathrm{eV}$ while the $\lambda_{\mathrm{h}}$ is fluctuating and increasing to $0.117 \mathrm{eV}$ as the basis sets become larger. The difference between $\lambda_{\mathrm{e}}$ and $\lambda_{\mathrm{h}}$ is narrowing

Table 6. Influence of basis sets on vertical and adiabatic ionization potentials $\left(I P_{v}\right.$ and $\left.I P_{a}\right)$, vertical and adiabatic electron affinities $\left(E A_{v}\right.$ and $\left.E A_{a}\right)$, and reorganization energies ( $\lambda_{h}$ and $\lambda_{e}$ ) for TPB by B3LYP method (in eV)

\begin{tabular}{ccccccc}
\hline Basis set & $I P_{\mathrm{v}}$ & $I P_{\mathrm{a}}$ & $E A_{\mathrm{v}}$ & $E A_{\mathrm{a}}$ & $\lambda_{\mathrm{e}}$ & $\lambda_{\mathrm{h}}$ \\
\hline 6-31G & 7.319 & 7.308 & 0.688 & 0.543 & 0.291 & 0.099 \\
6-31G* & 7.230 & 7.234 & 0.689 & 0.538 & 0.306 & 0.081 \\
6-311G & 7.547 & 7.545 & 0.390 & 0.241 & 0.300 & 0.096 \\
6-311G* & 7.460 & 7.468 & 0.373 & 0.222 & 0.306 & 0.083 \\
6-311G** & 7.485 & 7.481 & 0.349 & 0.197 & 0.306 & 0.100 \\
6-311+G* & 7.544 & 7.551 & 0.126 & -0.017 & 0.286 & 0.111 \\
6-311++G** & 7.573 & 7.516 & 0.088 & -0.052 & 0.285 & 0.117 \\
\hline
\end{tabular}

at the B3LYP/6-311++G** level. Considering the balance of accuracy and practicality, B3LYP/6-311G** were chosen to estimate these properties in this work. Table 7 lists all the $I P$, $E A$ and $\lambda$ values obtained at B3LYP/6-311G**. The $I P_{\mathrm{v}}$ and $I P_{\mathrm{a}}$ values are close to each other, while the $E A_{v}$ and $E A_{a}$ values not match as well as IP. As the electron-withdrawing groups were attached, both IP and absolute value of $E A$ have a remarkable increase comparing to $\mathbf{1}$ except for $\mathbf{3}$ and $\mathbf{4}$. $E A$ reflects the electron injection barrier and performance durability for $\mathrm{n}$-type OSC. The EA values of $\mathbf{1}$ are negative due to using different basis sets. ${ }^{[51]}$ The large $E A$ results in a low injection barrier and high air-stable performance. At the same time, 6, 7 and 8 are also close to this threshold $-2.80 \mathrm{eV}$. Hence, 6, 7 and 8 are expected to be quite stable as the electron transport materials. The mean that $-\mathrm{CN}$ substituent is more suitable to modify TPB than $-F$ group from the aspect of $E A$.

Table 7. Calculated vertical and adiabatic ionization potentials $\left(I P_{\mathrm{v}}\right.$ and $\left.I P_{\mathrm{a}}\right)$, vertical and adiabatic electron affinities $\left(E A_{\mathrm{v}}\right.$ and $\left.E A_{\mathrm{a}}\right)$, and reorganization energies $\left(\lambda_{\mathrm{h}}\right.$ and $\left.\lambda_{\mathrm{e}}\right)$ for TPB and its derivatives obtained by B3LYP/6-311G** method (in eV)

\begin{tabular}{ccccccc}
\hline Compd. & $I P_{\mathrm{v}}$ & $I P_{\mathrm{a}}$ & $E A_{\mathrm{v}}$ & $E A_{\mathrm{a}}$ & $\lambda_{\mathrm{e}}$ & $\lambda_{\mathrm{h}}$ \\
\hline 1 & 7.48 & 7.48 & -0.349 & -0.197 & 0.306 & 0.100 \\
2 & 8.08 & 7.92 & 0.250 & 0.428 & 0.375 & 0.315 \\
3 & 7.48 & 7.37 & -0.138 & 0.102 & 0.549 & 0.206 \\
4 & 8.06 & 7.58 & -0.178 & 0.0445 & 0.474 & 0.478 \\
5 & 8.14 & 8.11 & 0.422 & 0.672 & 0.532 & 0.137 \\
6 & 9.26 & 9.21 & 1.90 & 2.06 & 0.304 & 0.161 \\
7 & 8.64 & 8.60 & 1.46 & 1.59 & 0.282 & 0.0842 \\
8 & 7.86 & 7.85 & 1.67 & 1.90 & 0.449 & 0.151 \\
9 & 9.63 & 9.52 & 3.58 & 3.75 & 0.336 & 0.186 \\
\hline
\end{tabular}




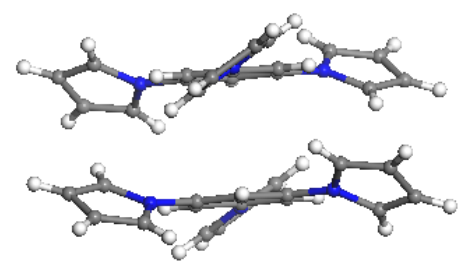

(a)

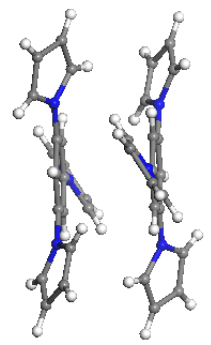

(b)

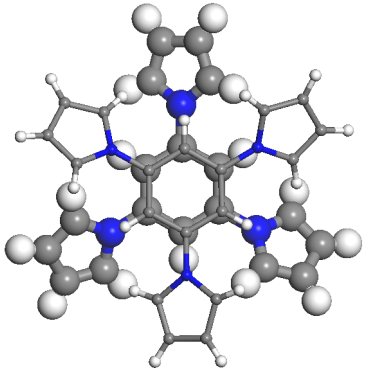

(c)

Figure 3. Molecular packing of dimer 1 from different perspectives.

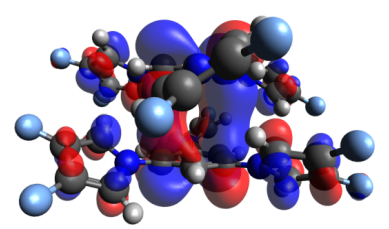

(a)

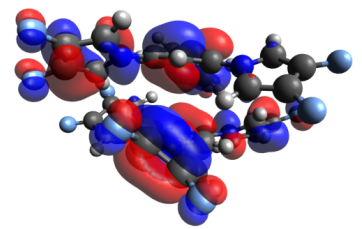

(b)

Figure 4. Topology surfaces of LUMOs (a) and HOMOs (b) for dimer 2 at WB97XD /6-31G level.

In Table 7, it can be noticed that $\lambda_{\mathrm{e}}$ for all molecules are larger than $\lambda_{\mathrm{h}}$ by 2 or 3 times. This is not good news for this searching new potential n-type OSC effort. According to equation (1), a large $H_{\text {eff }}$ would have great impacts on the final charge transfer mobility. In general, $-F$ substituent result in an obviously increasing on $\lambda_{\mathrm{e}}$ except for $\mathbf{8}^{[50,48]}$ and the introduced substituent groups can bring in more vibrational modes of geometric relaxation that will lead to $\lambda_{\mathrm{e}}$ increase from the aspect of normal-mode (MN). ${ }^{[17,48,53,54]}$ The triple bond of $-\mathrm{CN}$ which was connected into the original molecular $\pi$-conjugate system makes the conjugate system a further expanded. And the injected electron can locate on a wider space, this can help to decrease the $\lambda_{\mathrm{e}}$. The $\lambda_{\mathrm{e}}$ of $\mathbf{3 , 4 , 5 , 8}$ are too large to be a well-perspective $n$-type OSC. According to above analysis, $-\mathrm{CN}$ is more suitable than $-\mathrm{F}$ group to modify TPB to search new n-type OSC.

\section{Dimer Configurations}

Many previous works showed that the conjugated planar molecules adopt the face to face packing in their dimer. ${ }^{[49]}$ Here we assumed that the dimers of $\mathbf{2 - 9}$ and TPB have a similar face to face packing. The face to face dimers are optimized by using the DFT-D functional which contains dispersion force correction (-D). ${ }^{[55]} \omega B$ 97XD functional was used for the corrected evaluation of the energy of weak interaction. ${ }^{[25]}$ These dimers are constructed by using the interlaced parallel manner (as shown in Figure 3 ) to be the initial geometry. This stacking motif can offer the highest $H_{\text {eff }}$ among the dimers of TPB. Then all the constructed face to face dimers were optimized at $\omega B$ 97XD/6-31G level. The adjacent monomers in our predicted dimers overlap partly or fully, while the distance between adjacent parallel benzene rings are $3.42 \AA$, only $0.08 \AA$ larger than that in crystal. ${ }^{[56]}$ This means that the simulation at $\omega B 97 X D / 6$ $31 \mathrm{G}$ level can offer relative accurate dimer structure. The monomers in dimers $\mathbf{3}, \mathbf{4}, \mathbf{5}$ and $\mathbf{8}$ overlap partially in a face to face packing while $\mathbf{9}$ almost has no overlaps. All the others keep the precisely parallel structure. Considering the facts that the electron $H_{\text {eff }}$ of the dimers mainly estimates the interactive strength between the monomer LUMOs and the LUMOs of these monomers mainly located in the benzene rings as shown in Figure 4, we define that all hopping distance for electron $\left(r_{\mathrm{e}}\right)$ are the center of mass of the parallel benzene ring, while hole hopping distance $\left(r_{\mathrm{h}}\right)$ are the distance between the centers of mass of the two monomers as most works done. ${ }^{[48,55,57,58,59]}$ In Figure 5, $r_{\mathrm{e}}$ and $r_{\mathrm{h}}$ for all dimers were listed (in 9, the adjacent molecular benzene rings is not parallel and have no obvious benzene overlap anymore. Thus the $r_{\mathrm{e}}$ and $r_{\mathrm{h}}$ are neglected). All the substituted TPB dimers have a larger $r$ than TPB. And the $r$ (both $r_{\mathrm{e}}$ and $r_{\mathrm{h}}$ ) is becoming larger from $-\mathrm{F}$ substituent to $-\mathrm{CN}$. This phenomenon is consistent with the above $\theta$ analysis. 


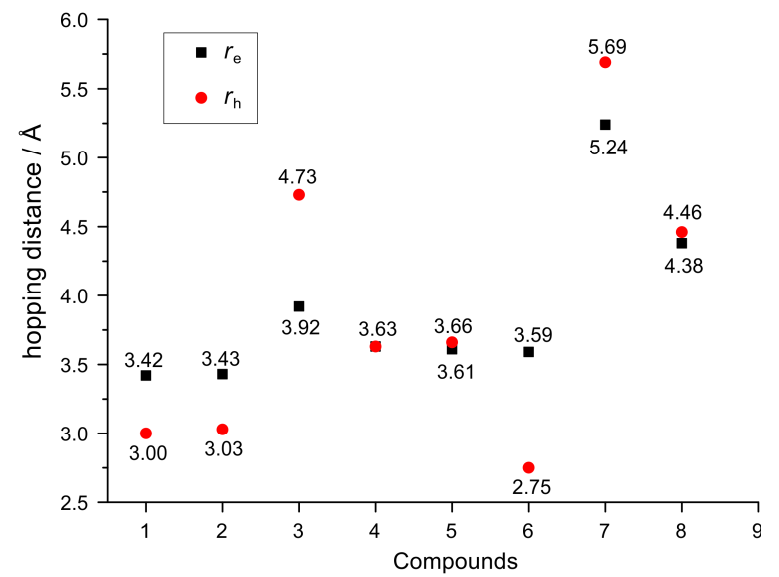

Figure 5. $r_{\mathrm{e}}$ and $r_{\mathrm{h}}$ of the optimized dimers.

\section{Charge Transfer Integrals, Diffusion Coefficients and Charge Mobility}

$H_{\text {eff }}$ is another importance index in the hopping model. In many simulations of single crystals, most hopping pathways have small $H_{\text {eff }}$ and these small $H_{\text {eff }}$ hardly contribute to the charge mobility. ${ }^{[5,59,60]}$ Thus, we studied the charge transport property by the face to face dimers. In this work, the PW91PW91 functional was used to evaluate the $H_{\text {eff }}$ as shown in Table 8. Actually, PW91PW91/6-31G(d,p) has been applied widely to calculate $H_{\text {eff }}$ and has been shown to be suitable for this calculation. ${ }^{[48,60-62]}$ Thus, in this work, the PW91PW91 was adopted to analysis. Our calculations reveal that the electron and hole $H_{\text {eff }}$ of 2 are $113.3 \mathrm{meV}$. Electron $H_{\text {eff }}$ of $\mathbf{2}$ is similar to $\mathbf{1}$ but much larger than the others compounds dimer. Figure 5 shows the topology surface of the LUMO and HOMO for $\mathbf{2}$. It can be seen from Figure 5 that the LUMO has obvious overlap interaction between the adjacent molecules while the HOMO scattered around the pyrrole rings and do not have direct interaction between the face to face monomers. Consequently, the $H_{\text {eff }}$ values for electron and hole are different greatly. This HOMO/LUMO topology surface of dimer $\mathbf{2}$ also is consist with its $H_{\text {eff }}$ value in Table 8 . According to our calculation, this series compounds all have larger electron $H_{\text {eff }}$ value than hole. The electron $H_{\text {eff }}$ of 5, 6, 7 and 8 are $33.2 \mathrm{meV}$, $33.5 \mathrm{meV}, 50.0 \mathrm{meV},-37.5 \mathrm{meV}$. Because of the $r_{\mathrm{e}}$ are increasing from $3.42 \AA$ for 1 to $3.66 \AA$, $3.59 \AA$, $5.24 \AA$ and $4.38 \AA$ respectaively, the electron $H_{\text {eff }}$ have a large decrease compared to the $113.3 \mathrm{meV}$. The substitutions of $-\mathrm{F}$ and $\mathrm{CN}$ groups make the electron and hole $H_{\text {eff }}$ decrease comparing to $\mathbf{1}$ especially for electron $H_{\text {eff. }}$ The charge transfer mobility can be calculated on the basis of $\lambda$ and $H_{\text {eff }}$ value. ${ }^{[48]}$ In Table 9, all the charge mobility (both hole and electron) values were listed. The charge mobilities of $\mathbf{1}$ are $0.433 \mathrm{~cm}^{2} \cdot \mathrm{V}^{-1} \cdot \mathrm{s}^{-1}$ (for electron) and $0.539 \mathrm{~cm}^{2} \cdot \mathrm{V}^{-1} \cdot \mathrm{s}^{-1}$ (for hole). However, the charge mobilities obtained from the dimer in crystal $1.14 \mathrm{~cm}^{2} \cdot \mathrm{V}^{-1} \cdot \mathrm{s}^{-1}$ (for electron) and
$0.341 \mathrm{~cm}^{2} \cdot \mathrm{V}^{-1} \cdot \mathrm{s}^{-1}$ (for hole). The discrepancy was caused by the different hopping distances in dimers obtained from theoretical optimization and from the crystal. But it seems reasonable when the different environment of the dimer (one is in the gas phase while the other is in solid single crystal) was taken into account. The $\mu_{\mathrm{h}}$ of $\mathbf{1}$ is also very high according to our calculation. Besides $\mathbf{1}$, the $\mu_{\mathrm{e}}$ of $\mathbf{2}$ and $\mathbf{7}$ are $0.198 \mathrm{~cm}^{2} \cdot \mathrm{V}^{-1} \cdot \mathrm{s}^{-1}$ and $0.279 \mathrm{~cm}^{2} \cdot \mathrm{V}^{-1} \cdot \mathrm{s}^{-1}$, respectively, which are relative larger than others. The $\mu_{\mathrm{e}}$ and $\mu_{\mathrm{h}}$ of $\mathbf{7}$ are almost identical $\quad\left(0.279 \mathrm{~cm}^{2} \cdot \mathrm{V}^{-1} \cdot \mathrm{s}^{-1}\right.$ and $0.277 \quad \mathrm{~cm}^{2} \cdot \mathrm{V}^{-1} \cdot \mathrm{s}^{-1}$, respectively). This balanced electron and hole transfer properties enable it to be a bipolar OSC. Substitutions in the $R_{2}$ and $R_{3}$ positions (Figure 1 ) of TPB greatly lower the coplanarity of TPB derivatives as seen the dihedral angle $(\theta)$ in Table 2. These bring in series negative effects in both reorganization energy and monomers packing. Thus $-\mathrm{F}$ and $\mathrm{CN}$ modification on TPB caused charge transfer property loss. In spite of this, TPB and some of its substituted derivatives (2 and 7) are likely to be promising OSC materials.

Table 8. Calculated effective charge transfer integrals (in meV) for all dimers at PW91PW91 /6-31G(d) level

\begin{tabular}{cccccc}
\hline Compd. & $\begin{array}{c}H_{\text {eff }} \\
\text { (hole) }\end{array}$ & $\begin{array}{c}H_{\text {eff }} \\
\text { (electron) }\end{array}$ & Compd. & $\begin{array}{c}H_{\text {eff }} \\
\text { (hole) }\end{array}$ & $\begin{array}{c}H_{\text {eff }} \\
\text { (electron) }\end{array}$ \\
\hline 1 & 39.1 & 113.3 & 6 & 22.1 & 33.5 \\
2 & 1.8 & 113.1 & 7 & 13.1 & 50.0 \\
3 & 0.1 & 21.7 & 8 & 12.2 & -37.5 \\
4 & -1.4 & 27.8 & 9 & 28.8 & -0.8 \\
5 & -5.3 & 33.2 & & & \\
\hline
\end{tabular}

Table 9. Electron and hole transfer mobility (in $\mathrm{cm}^{2} \cdot \mathrm{V}^{-1} \cdot \mathrm{s}^{-1}$ )

\begin{tabular}{cccccc}
\hline Compd. & $\mu_{\mathrm{h}}$ & $\mu_{\mathrm{e}}$ & Compd. & $\mu_{\mathrm{h}}$ & $\mu_{\mathrm{e}}$ \\
\hline $1^{\text {(a) }}$ & 0.341 & 1.14 & 5 & 0.008 & 0.004 \\
1 & 0.539 & 0.433 & 6 & 0.064 & 0.047 \\
2 & 0.000 & 0.198 & 7 & 0.277 & 0.279 \\
3 & 0.000 & 0.002 & 8 & 0.058 & 0.017 \\
4 & 0.000 & 0.005 & 9 & 0.215 & 0.000 \\
\hline (a) Dimer from X-ray crystal structure of TPB & &
\end{tabular}

\section{CONCLUSION}

The geometric, electronic, and charge transport properties of 1,3,5-tripyrrolebenzene (TPB) and its fluorinated and cyanated derivatives have been investigated by DFT calculations in combination with the Marcus hopping model. The results show that the electron-withdrawing groups cause somewhat geometrical deformation. The dihedral angle between peripheral pyrrole rings and center benzene 
ring increases especially in the case of cyanation. The introduction of fluorine and cyano group stabilizes the frontier molecular orbital and enhance the air stability. Fluorination increases the reorganization energy while cyanation does not. The $\lambda_{\mathrm{e}}$ values of these series compounds are larger than their $\lambda_{\mathrm{h}}$. Except for 9, all dimers adopt a face to face packing that is similar to TPB crystal or a slight slip away according to our dimer simulation, but the center-of-mass distance increases. The predicted quasione-dimensional electron mobilities of $\mathbf{1}, \mathbf{2}$ and 7 are $0.433 \mathrm{~cm}^{2} \cdot \mathrm{V}^{-1} \cdot \mathrm{s}^{-1}, 0.198 \mathrm{~cm}^{2} \cdot \mathrm{V}^{-1} \cdot \mathrm{s}^{-1}$ and $0.279 \mathrm{~cm}^{2} \cdot \mathrm{V}^{-1} \cdot \mathrm{s}^{-1}$, respectively. 6 and 7 show a bipolar-OSC performance. The electron injection barriers of $\mathbf{2}$ and $\mathbf{7}$ are smaller than TPB molecule. These outcomes denote that $\mathbf{1 , 2}$ and $\mathbf{7}$ are wellperformance $n$-type OSC.

Acknowledgment. The authors thank the National Science Foundation of China (No. 21372116) and the Project Funded by China Postdoctoral Science Foundation (No.2015M581802) for supporting this work.

\section{REFERENCES}

[1] H. E. Katz, Z. Bao, J. Phys. Chem. B 1999, 104, 671.

[2] M. L. Tang, T. Okamoto, Z. Bao, J. Am. Chem. Soc. 2006, 128, 16002.

[3] D. Braga, G. Horowitz, Adv. Mater. 2009, 21, 1473.

[4] J. Zaumseil, H. Sirringhaus, Chem. Rev. 2007, 107, 1296.

[5] A. Tsumura, H. Koezuka, T. Ando, Appl. Phys. Lett. 1986, 49, 1210.

[6] V. C. Sundar, J. Zaumseil, V. Podzorov, E. Menard, R. L. Willett, T. Someya, M. E. Gershenson, J. A. Rogers, Science 2004, 303, 1644.

[7] Y. Takahashi, T. Hasegawa, S. Horiuchi, R. Kumai, Y. Tokura, G. Saito, Chem. Mater. 2007, 19, 6382.

[8] O. D. Jurchescu, M. Popinciuc, B. J. van Wees, T. T. M. Palstra, Adv. Mater. 2007, 19, 688.

[9] J. Takeya, M. Yamagishi, Y. Tominari, R. Hirahara, Y. Nakazawa, T. Nishikawa, T. Kawase, T. Shimoda, S. Ogawa, Appl. Phys. Lett. 2007, 90, 102.

[10] K. Nakayama, Y. Hirose, J. Soeda, M. Yoshizumi, T. Uemura, M. Uno, W. Li, M. J. Kang, M. Yamagishi, Y. Okada, E. Miyazaki, Y. Nakazawa, A. Nakao, K. Takimiya, J. Takeya, Adv. Mater. 2011, 23, 1626.

[11] C. Foces-Foces, C. Fernández-Castaño, R. M. Claramunt, C. Escolástico, J. Elguero, J. Incl. Phenom. Macro. 1999, 33, 169.

[12] B. Ellman, R. J. Twieg, Phys. Rev. Lett. 2008, 100, 146401.

[13] Y. C. Cheng, R. J. Silbey, D. A. da Silva Filho, J. P. Calbert, J. Cornil, J. L. Brédas, J. Chem. Phys. 2003, 118, 3764.

[14] Z. Iqbal, A. P. Webb, S. Vepřek, Appl. Phys. Lett. 1980, $36,163$.
[15] M. J. Tauber, R. F. Kelley, J. M. Giaimo, B. Rybtchinski, M. R. Wasielewski, J. Am. Chem. Soc. 2006, 128, 1782.

[16] V. Coropceanu, J. Cornil, D. A. da Silva Filho, Y. Olivier, R. Silbey, J. L. Brédas, Chem. Rev. 2007, 107, 926.

[17] C. Zhao, Y. Guo, L. Guan, H. Ge, S. Yin, W. Wang, Synth. Metal. 2014, 188, 146.

[18] S. Chai, S. H. Wen, J. D. Huang, K. L. Han, J. Comput. Chem. 2011, 32, 3218.

[19] R. A. Marcus, Rev. Mod. Phys. 1993, 65, 599.

[20] N. S. Hush, J. Chem. Phys. 1958, 28, 962.

[21] J. L. Brédas, J. P. Calbert, D. A. D. S. Filho, J. Cornil, Proc. Natl. Acad. Sci. 2002, 99, 5804.

[22] I. V. Brovchenko, Chem. Phys. Lett. 1997, 278, 355.

[23] J. E. Norton, J. L. Brédas, J. Am. Chem. Soc. 2008, 130, 12377.

[24] M. Malagoli, J. L. Brédas, Chem. Phys. Lett. 2000, 327, 13.

[25] W. Senevirathna, C. M. Daddario, G. Sauvé, J. Phys. Chem. Lett. 2014, 5, 935.

[26] V. Coropceanu, M, Malagoli, D. A, da Silva Filho, N. E. Gruhn, T. G. Bill, J. L. Brédas, Phys. Rev. Lett. 2002, 89, 275503.

[27] D. L. Cheung, T. Alessandro, Phys. Chem. Chem. Phys. 2008, 10, 5941.

[28] W. Q. Deng, W. A. Goddard, J. Phys. Chem. B 2004, 108, 8614.

[29] M. M. Torrent, P. Hadley, S. T. Bromley, X. Ribas, J. Tarrés, M. Mas, E. Molins, J. Veciana, C. Rovira , J. Am. Chem. Soc. 2004, 126, 8546.

[30] Y. C. Chang, I. Chao, J. Phys. Chem. Lett. 2010, 1, 116.

[31] K. Sakanoue, M. Motoda, M. Sugimoto, A. S. Sakaki, J. Phys. Chem. A 1999, 103, 5551.

[32] K. Senthilkumar, F. C. Grozema, F. M. Bickelhaupt, L. D. A. Siebbeles, J. Chem. Phys. 2003, 119, 9809.

[33] G. García, M. Moral, J. M. Granadino-Roldán, A. Garzón, A. Navarro, M. Fernández-Gómez, J. Phys. Chem. C 2013, 117, 15.

[34] J. Cornil, V. Lemaur, J. P. Calbert, J. L. Brédas, Adv. Mater. 2002, 14, 726.

[35] Y. X. Zhang, X. Cai, Y. Z. Bian, X. Y. Li, J. Z. Jiang, J. Phys. Chem. C 2008, 112, 5148.

[36] A. M. Isa, Optoelectron. Adv. Mater. 2012, 6, 902.

[37] C. Suleyman, Optoelectron. Adv. Mater. 2009, 11, 280.

[38] A. Georgescu, G. Damache, M. A. Girtu, Optoelectron. Adv. Mater. 2008, 10, 3003.

[39] L. Horváthová, M. Dubecký, L. Mitas, I. Štich, Phys. Rev. Letts. 2012, 3, 053001.

[40] B. K. Ong, L. W. Kai, A. Ariffin, Synth. Metal. 2014, 195, 54.

[41] G. Zhang, C. B. Musgrave, J. Phys. Chem. A 2007, 111, 1554.

[42] A. A. Popov, L. Dunsch, J. Am. Chem. Soc. 2008, 130, 17726. 
[43] H. Wang, Y. He, Y. li, H. Su, J. Phys. Chem. A 2012, 116, 255.

[44] A. Irfan, J. Zhang, Y. Chang. Theor. Chem. Acc. 2010, $127,587$.

[45] L. Wang, Q. Li, Z. Shuai, L. Chen, Q. Shi, Phys. Chem. Chem. Phys. 2010, 12, 3309.

[46] J. Yin, Y. Hu, X. H. Ju, J. Mol. Model. 2014, 20, 2460.

[47] M. J. Frisch, G. W. Trucks, H. B. Schlegel, G. E. Scuseria, M. A. Robb, J. R. Cheeseman, G. Scalmani, V. Barone, B. Mennucci, G. A. Petersson, H. Nakatsuji, M. Caricato, X. Li, H. P. Hratchian, A. F. Izmaylov, J. Bloino, G. Zheng, J. L. Sonnenberg, M. Hada, M. Ehara, K. Toyota, R. Fukuda, J. Hasegawa, M. Ishida, T. Nakajima, Y. Honda, O. Kitao, H. Nakai, T. Vreven, J. A. Montgomery, Jr., J. E. Peralta, F. Ogliaro, M. Bearpark, J. J. Heyd, E. Brothers, K. N. Kudin, V. N. Staroverov, R. Kobayashi, J. Normand, K. Raghavachari, A. Rendell, J. C. Burant, S. S. lyengar, J. Tomasi, M. Cossi, N. Rega, J. M. Millam, M. Klene, J. E. Knox, J. B. Cross, V. Bakken, C. Adamo, J. Jaramillo, R. Gomperts, R. E. Stratmann, O. Yazyev, A. J. Austin, R. Cammi, C. Pomelli, J. W. Ochterski, R. L. Martin, K.Morokuma, V. G. Zakrzewski, G. A. Voth, P. Salvador, J. J. Dannenberg, S. Dapprich, A. D. Daniels, Ö. Farkas, J. B. Foresman, J. V. Ortiz, J. Cioslowski and D. J. Fox, Gaussian 09, Revision C.03, Gaussian, Inc., Wallingford, CT, 2010.

[48] C. B. Zhao, W. L. Wang, S. W. Yin, Y, Ma, New. J. Chem. 2013, 37, 2925.
[49] S. Chai, S. H. Wen, J. D. Huang, K. L. Han, J. Comput. Chem. 2011, 32, 3218.

[50] S. Chai, S. H. Wen, K. L. Han, Org. Electron. 2011, 12, 1806.

[51] H. X. Li, R. H. Zheng, Q. Shi, J. Phys. Chem. C 2012, 116, 11886.

[52] A. Ramírez-Solís, J. Comput. Chem. 2014, 2, 31.

[53] J. Cortes, H. Heitele, J. Jortner, J. Phys. Chem. 1994, 98, 2527.

[54] A. Kapturkiewicz, J. Herbich, J. Karpiuk, J. Nowacki, J. Phys. Chem. A 1997, 101, 2332.

[55] V. T. T. Huong, T. B. Tai, M. T. Nguyen, Phys. Chem. Chem. Phys. 2012, 14, 14832.

[56] K. Thallapally, K. Chakraborty, A. K. Katz, H. L. Carrell, S. Kotha, G. R. Desiraju, Cryst. Eng. Comm. 2001, 31, 1.

[57] A. M. Zhong, Y. Z. Bian, Y. X. Zhang, J. Phys. Chem. C 2010, 114, 3248.

[58] X. K. Chen, J. F. Guo, L. Y. Zou, A. M. Ren, J. X. Fan, J. Phys. Chem. C 2011, 115, 21416.

[59] L. J. Wang, P. Li, B. Xu, H. Y. Zhang, W. J. Tian, Org. Electron. 2014, 15, 2476.

[60] M. C. R. Delgado, K. R. Pigg, D. A. D. S Filho, N. E. Gruhn, Y. Sakamoto, T. Suzuki, R. M. Osuna, J. Casado, V. Hernández, J. T. L. Navarrete, N. G. Martinelli, J. Cornil, R. S. S. Carrera, V. Coropceanu, J. L. Brédas, J. Am. Chem. Soc. 2009, 131, 1502.

[61] Y. Song, C. Di, X. Yang, S. Li, W. Xu, Y. Liu, L. Yang, Z. Shuai, D. Zhang, D. Zhu, J. Am. Chem. Soc. 2006, 128, 15940.

[62] J. S. Huang, M. Kertesz, Chem. Phys. Lett. 2004, 390, 110. 